\title{
Surface Characterization of Nano-sized Star Block Copolymers for Antifouling Coatings on Water Purification Membranes
}

Jae-Young Cho ${ }^{1}$, Muhammad Amirul Islama ${ }^{2}$, Khalid Azyat ${ }^{1}$, Young-Hye La ${ }^{3}$, Mohtada Sadrzadeh ${ }^{2}$ and Andrew Myles ${ }^{1}$

${ }^{1}$ National Research Council CANADA - Nanotechnology Research Centre, Edmonton, Alberta, Canada,

${ }^{2}$ University of Alberta, Edmonton, Alberta, Canada, ${ }^{3}$ IBM Almaden Research Center, San Jose, California, United States

In recent years, there has been an increasing interest in nano-sized star block copolymers which have hydrophobic core and hydrophilic arms since they self-assemble into a thin and stable coating on various membrane surface increasing the antifouling properties of ultrafiltration membrane system [1-4]. However, their unique structure-property relationships with respect to the local surface characteristics (hydrophilicity/hydrophobicity) by varying the size of core and arms is not yet fully understood. In this study, star-shaped block copolymers with different sizes of core and arms was prepared to study the difference in local surface characteristics of star polymers. In order to understand the detail structural information of different sized star polymers, various cutting-edge microscopic characterization methods such as scanning transmission electron microscopy (STEM), transmission electron microscopy (TEM) and atomic force microscopy (AFM) were used. Especially, new phase mode AFM imaging method was developed for the illustration of local hydrophobicity/hydrophilicity on the surface of star polymer, and in-solution AFM imaging was performed to understand the actual arrangement of arms in water.

It is known that characterization of nano-sized star polymers (SPs) using electron microscopy (EM) is challenging since they are prone to being degraded by the strong electron beam. Therefore, the combination of optimized sample preparation and microscopic conditions are critical for successful characterization. In order to understand the detailed structure of SPs, three different star block copolymers (SP1, SP2 and SP3) which have different sizes of polystyrene (PS) core and polyethylene glycol (PEG) arms were synthesized (Figure 1a). For the EM studies, $0.02 \%$ of SPs solution were deposited on a carboncoated 400-mesh copper TEM grid, the excess solution was blotted after $10 \mathrm{~s}$. The staining of samples for STEM/TEM was performed by depositing one droplet of a $2 \%$ uranyl acetate solution for $120 \mathrm{~s}$. The grid was then blotted and dried in air. For AFM measurement of SPs, clean silica substrate $\left(1 \times 1 \mathrm{~cm}^{2}\right)$ was prepared and pretreated by UV-Ozone cleaner. Then, the samples were deposited by spin-coating at 2500 rpm for $30 \mathrm{~s}$ to remove the excessive precipitation from the surface of sample. These samples were dried in the air for a day.

STEM images were obtained at $30 \mathrm{kV}$ accelerating voltage and $30 \mu \mathrm{A}$ emission current on a ultra-high resolution Hitachi S-5500 cold field emission SEM. TEM imaging was carried out on Hitachi HT7700 TEM at $60 \mathrm{kV}$. Phase mode AFM images were obtained using a Veeco Instruments MultiMode Nanoscope IV AFM, equipped with an E scanner, along with high -resolution $(<1 \mathrm{~nm})$ soft tapping mode silicon tips which have low spring constants of $5 \mathrm{~N} / \mathrm{m}$. For in-solution AFM investigation, sample surface was merged in deionized water along with AFM tip which is attached with a fluid cell, and keep it for 2 hours to stabilize the sample surface. Tapping mode AFM tips which have low force constant $(2 \mathrm{~N} / \mathrm{m})$ and resonant frequency $(67 \mathrm{kHz})$ were used with low scan rate $(0.3 \mathrm{~Hz})$.

As shown in Figure 1b, STEM image of SPs shows the dark round shape of particles which are well dispersed on the surface of carbon film. Also, it is seen that the hydrophilic arms from STEM images of SPs, however the boundary between core and arms was not clearly distinguishable (bottom insets of Figure 
1b). Therefore, bright filed TEM images of SPs were taken (top insets of Figure 1b) and it clearly revealed its boundary between hydrophilic arms and hydrophobic core. Measured sizes of SPs (SP1, SP2 and SP3) were $20 \pm 2 \mathrm{~nm}, 21 \pm 3 \mathrm{~nm}$ and $28 \pm 4 \mathrm{~nm}$ respectively. For the final surface characteristic of SPs, it is important to understand that the arrangement of arms in the SPs (folded or stretched), especially SP3 which has a longer arms. From this TEM investment, it seems that longer arms in SP3 are partially stretched out toward the outside of SP cores.

To identify local hydrophilicity/hydrophobicity from SPs surface, the phase mode AFM imaging was performed since knowing the degree of hydrophilicity in each SPs is critical for understanding anti-fouling property of SPs coated water membrane. During tapping mode AFM, the phase shift is derived from the difference in phase angle between the freely oscillating cantilever in air and the cantilever oscillation during scanning, and it is very sensitive to local variations in the materials properties [5]. As shown in Figure 2a, SPs on silica surface were scanned by high-resolution silicon tip and these phase mode AFM images revealed their hydrophilic rich area (bright yellow color) and hydrophobic core (dark brown color). To make these findings clear, the root-mean-square (RMS) values of phase shift were calculated, and the highest value was found in SP3. In addition, in-solution AFM was performed to see the structural changes, especially the arrangement of hydrophilic arms of SPs in water. As shown in Figure 2b, height mode AFM images of SPs in water show that it sticks to silica surface and hold its structure very well, except SP3 which has a longer arms and bigger core. In SP3, it seems that longer arms are folded into near SP core area when it is dried, however, these longer arms are stretched out when it is in water, as indicated by arrows (Figure 2b).

In summary, three star polymers which have different sizes of core and arms were successfully characterized by STEM, TEM, and AFM (phase mode in-air and in-solution). These star polymers' sizes ranged from 18 to $32 \mathrm{~nm}$ which is confirmed by TEM. The local hydrophilicity/hydrophobicity of SPs were visualized by phase mode AFM imaging: the highest RMS value was found in SP3 as expected. Insolution AFM study revealed the arrangement of arms in SPs is different when it is dried or in water. In the near future, these various types of star polymers will be used for coating of membrane and characterized by in-situ AFM and cryo-SEM/FIB [6].

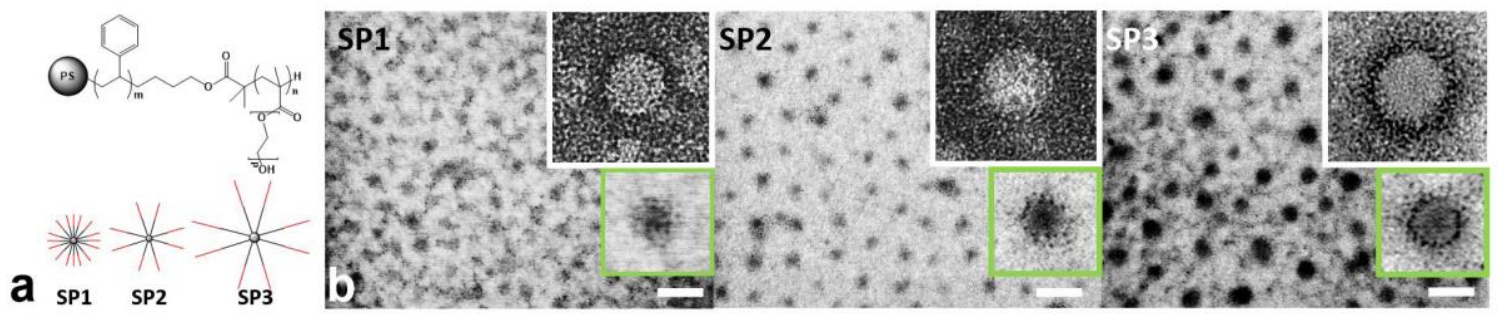

Figure 1. SP2 SP3 Figure 1. Three different star polymers (SPs): a) molecular structure and schematic diagram, b) STEM and TEM (top insets) images. Scale bar $=50 \mathrm{~nm}$.
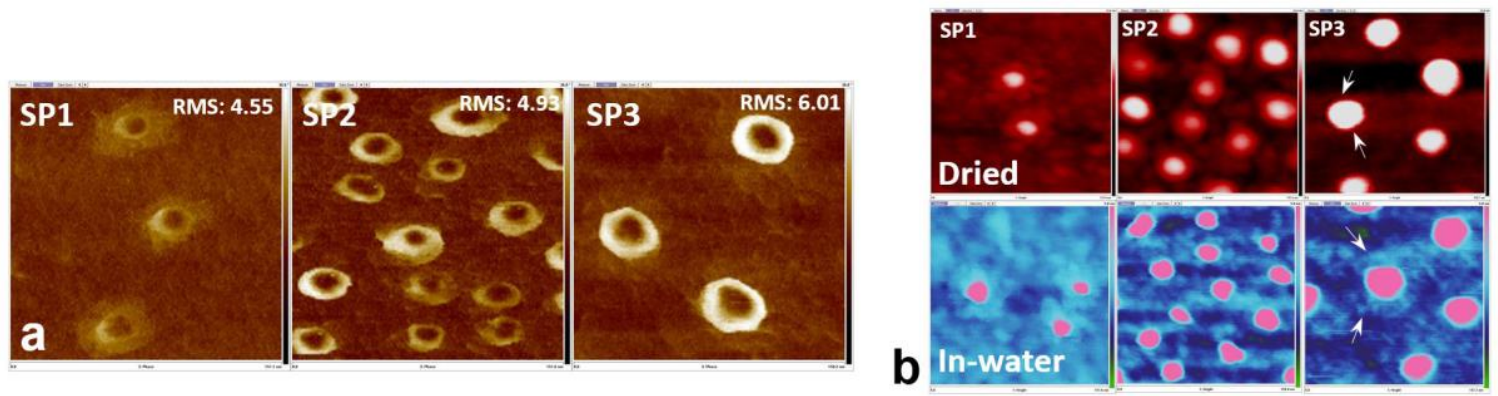
Figure 2. AFM images of star polymers (SPs): a) phase mode in air and b) height mode in water.

\section{References}

[1] JM Ren et al., Chem. Rev., 116, 12 (2016), p. 6743.

[2] DG Kim et al., J. Mater. Chem., 22 (2012), p. 8654.

[3] Q Fu et al., Macromol. Rapid Commun. 33 (2012), p. 2109.

[4] J Diep et al., Polymer 103 (2016), p. 468.

[5] K. Boussu et al., J. Colloid Interface Sci. 286 (2005), p. 632.

[6] The authors acknowledge funding from the IBM Alberta Centre for Advanced Studies (CAS), IBM Research-Almaden, Alberta Innovates (AI), National Research Council CANADA, University of Alberta and Government of Alberta 